Disponível em

http://www.anpad.org.br/rac

RAC, Rio de Janeiro, v. 19, n. 1, art. 3, pp. 45-64, Jan./Fev. 2015

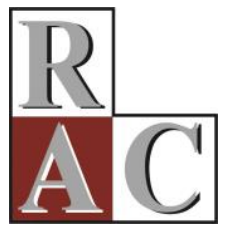

\title{
Mercado Voluntário de Carbono: Análises de Cobenefícios de Projetos Brasileiros
}

\author{
Voluntary Carbon Markets: Analysis of Co-benefits of Brazilian Projects
}

Danielle Soares Paiva

Universidade Federal da Bahia - UFBA

Luz Garcia Fernandez

Universidade Politécnica de Madrid - UPM

Andréa Cardoso Ventura

Universidade Federal da Bahia - UFBA

Guineverre Alvarez

Universidade Federal da Bahia - UFBA

José Célio Silveira Andrade

Universidade Federal da Bahia - UFBA 


\title{
Resumo
}

Este estudo apresenta resultados parciais de uma pesquisa que teve por objetivo identificar e analisar os cobenefícios em prol do desenvolvimento sustentável de projetos do mercado voluntário de carbono no Brasil para além da redução de gases de efeito estufa. Para tanto, foram realizadas pesquisas exploratórias, análise documental e estudos de casos ilustrativos, tendo como base a matriz analítica construída a partir de estudos da United Nations Framework Conventions on Climate Change (UNFCCC) sobre cobenefícios oriundos do mercado regulado de carbono. Os resultados revelaram que dois dos quatro projetos analisados apresentaram maiores avanços na promoção do desenvolvimento sustentável, o que difere dos achados da UNFCCC (2011) e de Boyd et al. (2009). O resultado parcial sinaliza maior potencial do mercado voluntário em comparação ao mercado regulado de carbono para contribuição ao desenvolvimento sustentável em âmbito local, dada sua maior flexibilidade e a diversidade de atores, assim como a exigência, por parte de alguns padrões de certificação, da demonstração do alcance dos cobenefícios declarados. Ademais, notou-se que alguns cobenefícios estimulam a ocorrência de outros nas localidades onde se desenvolvem e que os projetos com melhores contribuições estavam registrados em padrões de certificação que exigem o atendimento a indicadores que vão além da redução de emissão de gases de efeito estufa.

Palavras-chave: desenvolvimento sustentável; cobenefícios; mercado de créditos de carbono; mercado voluntário de carbono; Brasil.

\begin{abstract}
This study presents partial results of a research that aims to examine the co-benefits of sustainable development of the voluntary carbon market in Brazil. Therefore, we carried out exploratory research, document analysis and illustrative case studies, based on the analytical matrix constructed from the United Nations Framework Conventions on Climate Change (UNFCCC) studies on co-benefits from the regulated carbon market. The results presented showed that two of the four projects had greater advances in promoting sustainable development, contradicting the results of the UNFCCC (2011) and Boyd et al. (2009) in their studies on the regulated market. The achieved result confirms the major potential market for voluntary contribution to the sustainable development at the local scale, given greater flexibility and diversity of actors. Moreover, it was found that some co-stimulate other benefits in the local communities where they were developed, besides the more advanced projects that were registered in the International Standards that require the attendance of indicators that go beyond the reduction of greenhouse gases.
\end{abstract}

Key words: sustainable development; co-benefits; voluntary carbon market; carbon market; Brazil. 


\section{Introdução}

A intensificação das alterações e variações climáticas causadas pelo aquecimento global é apontada como uma ameaça às condições de vida da população mundial, com variações na amplitude de seus efeitos (Blois, Zarnetske, Fitzpatrick, \& Finnegan, 2013; Seroa da Motta, Hargrave, Luedemann, \& Gutierrez, 2011). Cientistas têm apontado que substâncias e processos naturais e antrópicos são vetores dessas alterações, mas que, no último caso, elas têm se acentuado e estão relacionadas principalmente ao aumento das emissões de gases de efeito estufa (GEE), entre estes o carbono (Intergovernmental Panel on Climate Change [IPCC], 2013).

Embora existam correntes céticas quanto a esses argumentos (ver Andrew, 2011; Priem, 2013; Scafetta, 2010; Soon, 2007, entre outros), iniciativas colaborativas internacionais têm sido empreendidas com o intuito de mitigar os impactos humanos ao clima. Um destaque é o Protocolo de Kyoto - cuja vigência foi iniciada em 2005 com adesão de 175 nações, um acordo multilateral interpaíses que adota instrumentos de mercado para auxiliar seus signatários a reduzirem suas emissões de GEE ou adotarem medidas de compensação (Organização das Nações Unidas [ONU], 2012).

Entre esses instrumentos, o Mecanismo de Desenvolvimento Limpo (MDL) é o que permite a participação de países em desenvolvimento ou em processo de industrialização (como o Brasil) no esquema global. Em resumo, a mitigação das emissões é contabilizada em cada projeto MDL, gerando créditos de carbono, que são, posteriormente, comercializados em um ambiente institucional denominado mercado regulado de carbono, com regras estabelecidas e monitoradas pelo sistema ONU (Corbera, Estrada, \& Brownet, 2009). Além da redução de emissão de GEE, as possibilidades do MDL vão mais além, buscando o investimento em tecnologias mais limpas e também a geração de cobenefícios nos países que os hospedam (Souza, Paiva, \& Andrade, 2011).

Os cobenefícios têm sido associados ao desenvolvimento sustentável (Olhoff, Markandya, Halsnaes, \& Taylor, 2004; Olsen \& Fenhann, 2008) e podem ser entendidos como ganhos que vão além da redução das emissões de GEE e observados em vários contextos, tais como a melhoria na qualidade do ar e da água, intensificação na preservação do solo, proteção contra enchentes, geração de energia elétrica para áreas rurais ou remotas e aumento nas oportunidades de emprego (Boyd et al., 2009; Castro \& Michaelowa, 2010). Assim, seriam uma estratégia ganha-ganha para gerar desenvolvimento para o país proponente e benefícios climáticos em uma única medida (Miyatsuka \& Zusman, 2012). A cada dia, novas pesquisas vêm afirmando o potencial de projetos para além da mitigação de GEE, tendo-os como estratégias importantes para a redução da pobreza (Anderson, 2011; Crowe, 2013) e como mecanismos a serem considerados para a garantia de desenvolvimento em âmbito local, mesmo frente a um cenário de mudanças climáticas (Fernández, Sota, Andrade, Lumbreras, \& Mazorra, 2014; Subbarao \& Lloyd, 2011).

Além do mercado regulado, existem os mercados voluntários de carbono em que os projetos, assim como o MDL no mercado regulado, são implementados em nações em desenvolvimento e seus créditos são comercializados após contabilização. Contudo, no voluntário, há uma maior amplitude de atores envolvidos (tanto implementadores quanto compradores), o que o torna um instrumento de mercado mais abrangente (Harris, 2007). Também nessa plataforma há o duplo objetivo de redução de emissão de GEE e geração de cobenefícios (Boyd et al., 2009; Peters-Stanley \& Yin, 2013), mas sua estrutura é menos burocrática que a da ONU (Taiyab, 2005). Essas razões contribuem para o crescimento do mercado voluntário em volume de projetos nos últimos anos: de $97 \mathrm{MtCO}_{2}$, em 201,1 para 101 $\mathrm{MtCO}_{2}$, em 2012 (Peters-Stanley \& Yin, 2013).

Críticas têm sido feitas aos resultados alcançados pelo Protocolo de Kyoto, como relativa concentração geográfica e setorial dos projetos - que privilegia regiões mais ricas - e o uso de tecnologias de caráter mais corretivo e de baixo conteúdo de inovação tecnológica (Boyd et al., 2009; Bozmoski, Lemos, \& Boyd, 2008) e também se questionam contribuições à promoção do desenvolvimento sustentável e melhoria nas condições de vida local a partir de cobenefícios (Cosbey et al., 2005; Orford, Raubenheimer, \& Kantor, 2004). 
Em meio às incertezas de continuidade e efetividade do Protocolo após 2017 (Benessaiah, 2012), o mercado voluntário se mostra uma via possível e esperançosa à continuidade das iniciativas já existentes e ao surgimento de novas. Enquanto estudos exploram a contribuição do MDL para a geração de cobenefícios (Newell, Jenner, \& Baker, 2009; Sutter \& Parreño, 2007; e destacadamente United Nations Framework Conventions on Climate Change [UNFCCC], 2011), que estudou 3.864 projetos em todo o mundo), poucos miram no mercado voluntário (ver abordagens em Guigon, Bellassen, \& Ambrosi, 2009; MacKerron, Egerton, Gaskell, Parpia, \& Mourato, 2009; Merger, Dutschke, \& Verchot, 2011), ou novas abordagens que buscam comparar os cobenefícios gerados pelo MDL em contraposição a projetos do mercado voluntário e outros tipos de projetos de mitigação (a exemplo de Crowe, 2013 e Fernández, 2014).

Assim, busca-se diminuir uma lacuna existente na literatura ao identificar e analisar quais os cobenefícios gerados pelos projetos brasileiros de redução de gases de efeito estufa no mercado voluntário de carbono. Devido à natureza multifacetada do tema e à necessidade de fornecer informações básicas antes de embarcar nas análises, este artigo traz uma breve descrição do mercado voluntário e da escolha da abordagem de cobenefícios utilizada. A estratégia metodológica consistiu na realização de estudos de casos ilustrativos de quatro projetos e na triangulação desses dados com o referencial teórico e a matriz analítica da UNFCCC (2011). Os resultados da pesquisa são sintetizados em um quadro analítico-descritivo que apoia as discussões e considerações finais.

Inteirar-se dessa realidade permite pensar em melhores oportunidades para explorar esse mercado em crescimento e, especialmente, os ganhos ambientais, sociais e econômicos a ele associados. No mesmo sentido, expor desconhecidas lacunas ou novos cenários contribui para a criação ou fortalecimento de estratégias locais e nacionais de combate às mudanças climáticas aliadas ao desenvolvimento sustentável. Entre essas contribuições pretendidas neste artigo, acredita-se ainda que o estímulo ao debate a partir de experiências reais pode aproximar ambientes ainda distantes: o acadêmico, o político e o campo.

\section{O Mercado Voluntário de Carbono}

O mercado de carbono - que engloba as vertentes Regulada e Voluntária - pode ser definido como a "compra e venda de licenças para emissões (direito de poluir) ou reduções de emissões (offsets) que foram respectivamente ou distribuídos por um órgão regulatório ou gerados por projetos de redução de emissões de GEE" (Peters-Stanley \& Yin, 2013, p. 6).

O mercado voluntário (MV) opera de forma alternativa e similar ao mercado regulado, também, negociando créditos originados de atividades de redução de GEE em países em desenvolvimento/industrialização (Capoor \& Ambrosi, 2006), entre elas: (a) as com metodologias de pequena escala não viáveis, do ponto de vista econômico, no mercado regulado; (b) as que não atendem a critérios estabelecidos pelo MDL e; (c) as que já computaram créditos retroativos, ou seja, créditos computados antes mesmo de seu registro (Kollmuss, Zink, \& Polycarp, 2008).

Uma particularidade do MV são os Padrões de Certificação por terceiros, que estipulam regras de implementação e operação dos projetos (no regulado, as regras são estipuladas pela ONU, por meio da Convenção Quadro das Nações Unidas sobre Mudança do Clima) (Nussbaumer, 2009). Esses padrões têm atuação destacada no MV por auditarem e monitorarem periodicamente as atividades certificadas a fim de verificar conformidades e/ou desvios e, assim, conferir maior confiabilidade a essas iniciativas (MacKerron et al., 2009; Souza et al., 2011). Isso se mostra importante dada a existência de falhas no setor (características de mensuração, fiscalização, contabilização das reduções de emissões, entre outras, importantes quando se trata de mercado de offset) que impactam na credibilidade dos créditos negociados (Bayon, Hawn, \& Hamilton, 2009; Hoffman, 2004). 
Guigon, Bellassen e Ambrosi (2009) classificam os padrões em duas categorias: (a) "Padrões básicos de carbono" (p. 4), que certificam métodos de contabilidade de carbono e garantem que cada crédito emitido corresponda a uma redução de emissões de uma tonelada de CO2 e; (b) "Padrões de carbono de múltiplos benefícios" (p. 4), que também incluem aspectos ambientais e sociais mais amplos. Entre os últimos, podem ser citados: Brasil Mata Viva Standard; CarbonFix Standard; Climate, Community \& Biodiversity Standard (CCB); Gold Standard (GS); Panda Standard; Plan Vivo Standards e Social Carbon. Em alguns destes, como o Social Carbon e Gold Standard, são elencados determinados indicadores específicos para mensuração desses benefícios. Para Peters-Stanley e Yin (2013) e Thomson Reuters (2013), há evidências de que projetos com essa natureza tendem a ser mais valorizados e os valores de seus créditos mais elevados.

Além dessas diferenças, não se observam contrastes significativos entre os projetos implementados nas duas vertentes do mercado de carbono em relação aos seus objetivos últimos (redução de GEE e benefícios ao desenvolvimento sustentável) (Guigon et al., 2009), o que possibilita elaboração de algumas análises comparativas entre as mesmas.

Assim, foram mapeados, nesta pesquisa, 170 projetos brasileiros que integram o MV e, de acordo com Simoni (2009) e Kollmuss, Zink e Polycarp (2008), são de pequena escala, já que constituem atividades de energia renovável (capacidade de até 15 megawatts) ou que resultam em reduções de emissões menores ou iguais a $60 \mathrm{tCO}_{2}$ eq por ano (Ministério da Ciência e Tecnologia [MCT], 2011).

A Figura 1 ilustra a distribuição desses 170 projetos certificados/registrados por escopo setorial no Brasil, apontando para os mais representativos (troca de combustível proveniente de mata nativa, suinocultura e troca de combustível fóssil) e para os menos (reflorestamento, energia renovável, eficiência energética e resíduos).

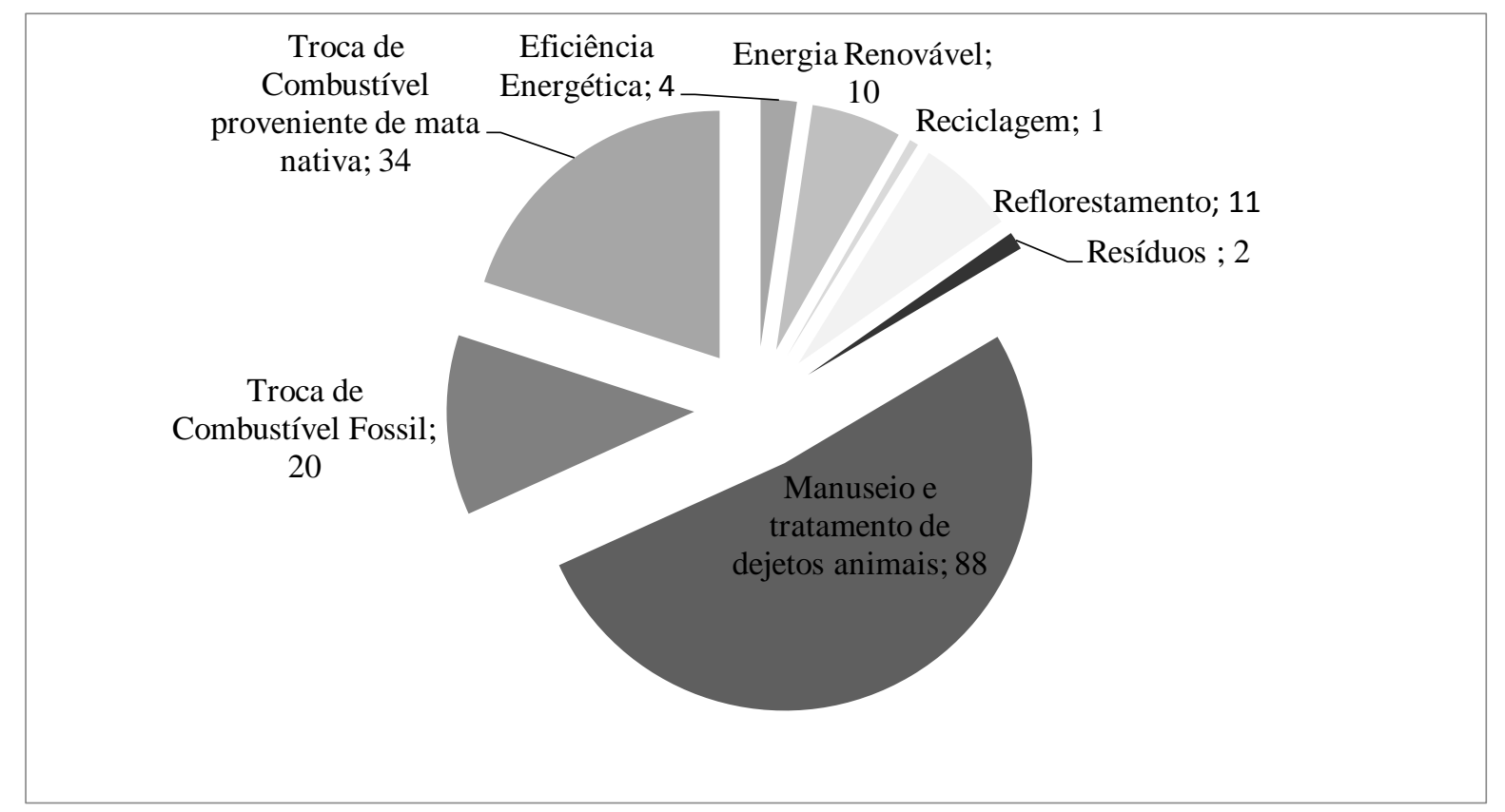

Figura 1. Número de Projetos Brasileiros de Redução de Gases de Efeito Estufa Registrados e Distribuídos por Escopo Setorial.

Fonte: Elaborado pelos autores.

Observa-se, na Figura 1, que os três escopos setoriais mais representativos (manuseio e tratamento de desejos de animais; troca de combustível e reflorestamento) concentram mais de $70 \%$ dos projetos de redução de emissão de créditos de carbono no Brasil, sendo que o maior escopo concentra mais que $50 \%$ deles, fazendo com que o mercado voluntário brasileiro reflita sobre as características dos projetos do escopo de manuseio e tratamento de dejetos. 


\section{A Abordagem de Indicadores de Cobenefícios para o Desenvolvimento Sustentável no Mercado de Carbono}

A abordagem de cobenefícios em mudanças climáticas pode ser genericamente entendida como "políticas e medidas que visam atender simultaneamente às necessidades dos países em desenvolvimento, além de reduzir as emissões de gases de efeito estufa (GEE)" (Overseas Environmental Cooperation Center [OECC], 2009, p. 1). Contudo não tem sido fácil mensurar o desenvolvimento sustentável (DS) a partir de cobenefícios, dado que, até o momento, não há um consenso sobre ferramentas e métodos para determinação e avaliação dos mesmos (e seus intrínsecos critérios e indicadores), o que abre espaço para uma diversidade de instrumentos de medição e interpretação (Boyd et al., 2009; Jung, 2006). Não obstante esta dificuldade de mensuração é crescente o número de pesquisas que vêm afirmando a possibilidade de que projetos de mitigação promovam desenvolvimento local ao adotar estratégias como a aquisição de matéria-prima e contratação de mão de obra local, e outros instrumentos de redução de pobreza e melhoria de qualidade de vida (Crowe, 2013; Subbarao \& Lloyd, 2011).

No mercado regulado, os cobenefícios estão relacionados, em sua maioria, aos parâmetros determinados pela Autoridade Nacional Designada, uma organização responsável por avaliar se projetos de MDL auxiliarão o país anfitrião na realização de seus objetivos de desenvolvimento sustentável (UNFCCC, 2011). Em geral, esses parâmetros incluem ao menos três dimensões da sustentabilidade (econômica, social e ambiental) e atendem a um contexto específico, baseado em prioridades nacionais e institucionais, nas demandas do mercado e na participação (ou não) dos interessados em vários níveis.

Buscando superar essas dificuldades, a UNFCCC (2011) realizou estudos para avaliar o quanto um projeto de MDL contribui para o DS, elencando indicadores que apontam a natureza das mesmas. Trata-se de uma proposta inovadora, que procura suprir as deficiências detectadas por alguns autores (a exemplo de Olsen \& Fenhann, 2008; Sutter \& Parreno, 2007).

Os 15 indicadores trazidos pela UNFCCC (2011) cobrem três dimensões do DS utilizadas cumulada ou alternadamente em outros estudos (Alexeew et al., 2010; Boyd et al., 2009; Fernández, Lumbreras, Borge, \& Cobo-Benita, 2011; Olsen, 2007; Olsen \& Fenhann, 2008): desenvolvimento econômico, proteção ambiental e desenvolvimento social. Além de harmonizar em uma mesma matriz elementos de características e origens variadas com indicadores de escala nacional e local, já reconhecidos em trabalhos prévios, seu destaque está na amplitude dos dados coletados a partir dos documentos de concepção dos projetos, num total de 3.864 iniciativas globalmente distribuídas.

Na Figura 2, apresentam-se os resultados obtidos a partir da avaliação dos projetos de mecanismo de desenvolvimento limpo (MDL), feita segundo indicadores estabelecidos pelo UNFCCC (2011). No referido estudo, os resultados apontaram a ocorrência mais frequente de criação de emprego (23\%) e redução do ruído, odores, poeira ou poluição (17\%), o que corrobora os achados de Olsen e Fenhann (2008), que encontraram resultados similares em sua análise de projetos de MDL. Para esses autores, a geração de emprego foi o impacto mais provável, seguido da contribuição para o crescimento econômico e melhor qualidade do ar. 


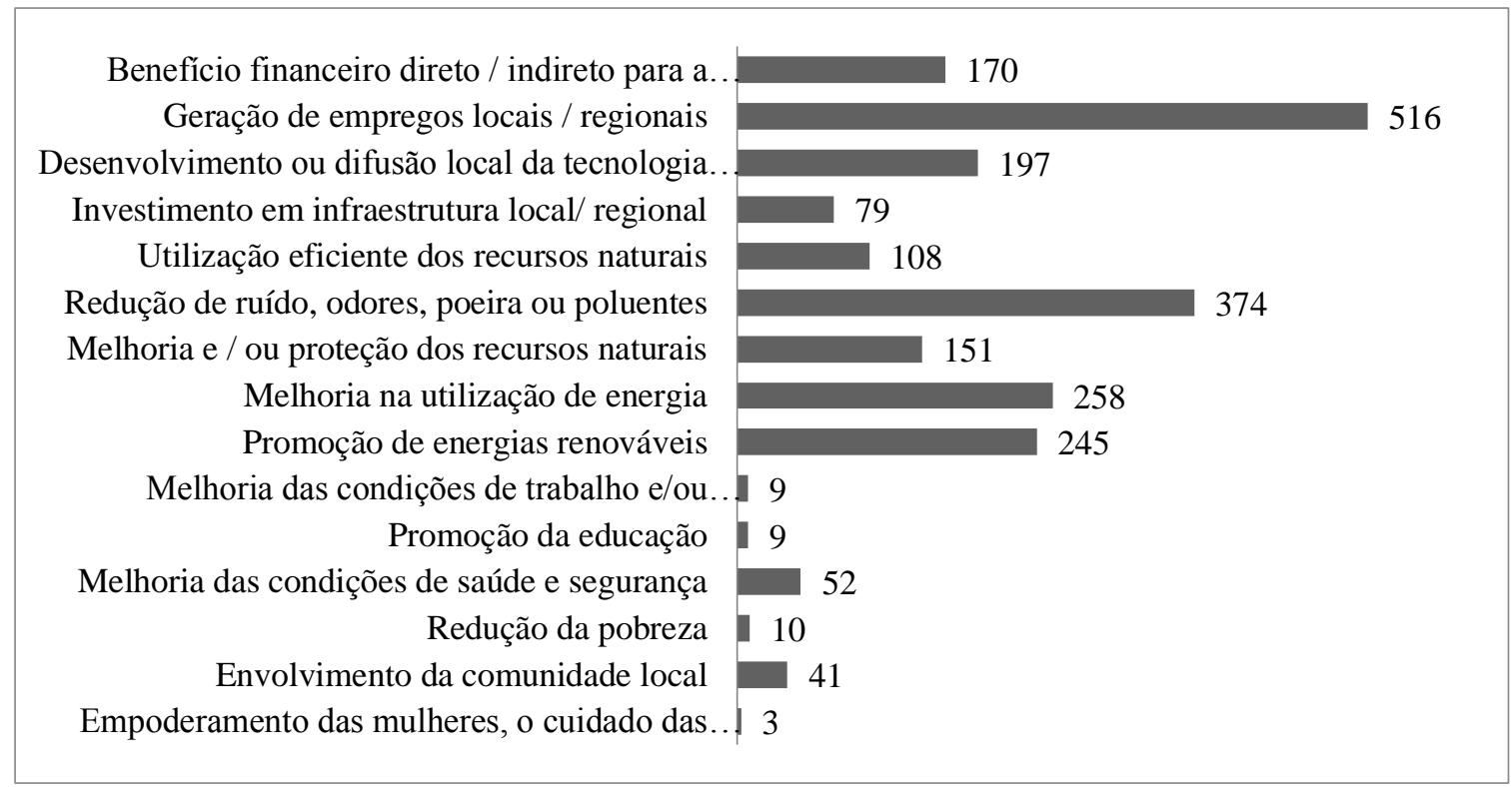

Figura 2. Número de Projetos de MDL por Cobenefícios Avaliados pelo UNFCCC. Fonte: United Nations Framework Conventions on Climate Change. (2011). Benefits of the clean development mechanism 2011. Retrieved from https://cdm.unfccc.int/about/dev_ben/ABC_2011.pdf

Ainda na pesquisa da UNFCCC (2011), observa-se que a maioria dos projetos de MDL analisados declara mais de um cobenefício, todavia nenhum deles é anunciado em mais de $25 \%$ dos projetos. O mais citado foi melhoria e/ou proteção dos recursos naturais (36\%), destacadamente em projetos de florestamento e reflorestamento. Há uma tendência de redução do ruído, odores, poeira ou poluentes (de $12 \%$, em 2005, para 21\%, em 2011), assim como para uma redução de utilização eficiente dos recursos naturais, com resultados de 7\% (2007) para 1\% (2011). As maiores contribuições sociais são reivindicadas por projetos de gás industrial, principalmente por meio do envolvimento da população local e promoção da educação. Na dimensão econômica, criação de emprego é o mais proeminente.

Por fim, a mesma investigação encontrou que os cobenefícios declarados não mudaram muito ao longo do tempo, sendo, no entanto, sempre diversificados. Foi observado também que, diante das inúmeras reivindicações declaradas nos Documentos de Concepção dos Projetos de MDL, há uma forte evidência de contribuição para o DS no país de acolhimento (o mesmo encontrou Castro \& Michaelowa, 2010).

Boyd et al. (2009), contudo, encontraram resultados diferentes e perceberam que, anos após a implementação do MDL, o objetivo de promover o DS no país anfitrião nem sempre foi atendido. Os autores atribuem isso à falta de clareza na definição do DS pela Autoridade Nacional Designada - o que dificulta ou impede a mensurabilidade dos mesmos - que, muitas vezes, está interessada apenas nos recursos financeiros provenientes dos projetos. Bumpus e Cole (2010) alertam que se deve exigir transparência e análises imparciais nos relatórios de acompanhamento, verificação e validação dessas iniciativas.

Comumente, estudos têm relacionado cobenefícios ao uso dos Padrões de Certificação no mercado voluntário (Drupp, 2011; MacKerron et al., 2009; Nussbaumer, 2009; Wood, 2011) e isso a um maior potencial de contribuição ao DS (Kollmuss et al., 2008; Taiyab, 2005). Crowe (2013) verificou que o uso dos padrões está também associado a um alto nível de envolvimento das partes interessadas, bem como à implementação de projetos por instituições sem fins lucrativos, governo ou organizações intergovernamentais.

Wood (2011) analisou como positivas as contribuições ao desenvolvimento local de atividades vinculadas a Gold Standard e Climate, Community and Biodiversity Standard. Merger, Dutschke e Verchot (2011) estudaram os mais populares esquemas de certificação para performance ambiental e social de projetos de reflorestamento e desmatamento evitado no MV e encontraram que, embora os 
padrões não possuam critérios homogêneos para cobenefícios, ainda assim, as iniciativas por eles certificadas contribuem para a melhoria das condições ambientais e sociais das comunidades.

No contexto brasileiro, há investigações baseadas em estudos de caso único que demonstram que o uso de Padrões de Certificação do MV apresenta grande potencial de gerar cobenefícios em escala local. Ventura, Fernández, Andrade e Lumbreras (2012), por exemplo, ao estudarem Fogões Eficientes certificados por Gold Standard, identificaram relevantes cobenefícios sociais e ambientais. Paiva, Gomes, Fernández e Andrade (2014) encontraram cobenefícios (destacadamente locais) em um projeto de reflorestamento certificado pelo Climate, Community and Biodiversity Standard (CCB).

No entanto mais estudos e verificações no Brasil ainda são necessários para melhor conhecer e delimitar as possibilidades de ganhos ambientais, sociais e econômicos das iniciativas de redução de GEE no mercado voluntário de carbono.

\section{Procedimentos Metodológicos}

Os procedimentos metodológicos do trabalho de pesquisa envolveram três fases, a primeira de caráter exploratório, a segunda a análise documental e terceira com estudos de caso.

A primeira fase do trabalho envolveu a coleta de dados secundários em documentos oficiais dos projetos de redução de GEE estudados e também nas literaturas nacional e internacional. Essa fase teve caráter exploratório e concentrou-se em mapeamento e levantamento documental dos Documentos de Concepção dos Projetos brasileiros de redução de GEE no MV. Esses documentos, desenvolvidos pelos implementadores e obrigatórios em processos de certificação, fornecem detalhadamente informações quanto às características e aos objetivos desses empreendimentos. Também foram pesquisadas outras fontes de informação, como relatórios de auditoria, corporativos e técnicos, caracterizando, portanto, uma análise ex ante. A revisão de literatura se deu em bases nacionais e internacionais por meio de consultas a livros, periódicos e sites institucionais dos principais padrões de certificação de créditos de carbono (VCS, CCB, Gold Standard, Social Carbon, CCX, etc.). Essa primeira fase envolveu também a escolha, a partir da revisão da literatura, do framework utilizado para a análise dos cobenefícios dos projetos nas perspectivas social, ambiental e econômica e que proporcionasse uma comparação com o mercado regulado. Assim, optou-se por utilizar, com pequena adaptação (tradução da tabela do inglês para português), o framework da UNFCCC (2011), conforme Tabela 1:

Tabela 1

\section{Framework da Pesquisa}

\begin{tabular}{llll}
\hline Conceito & Dimensão & Componente & Indicadores \\
\hline $\begin{array}{l}\text { Cobenefícios do } \\
\begin{array}{l}\text { Mercado de } \\
\text { Carbono Voluntário }\end{array}\end{array}$ & $\begin{array}{l}\text { Desenvolvimento } \\
\text { Sustentável }\end{array}$ & $\begin{array}{l}\text { Desenvolvimento } \\
\text { Econômico }\end{array}$ & $\begin{array}{l}\text { Benefício financeiro direto/indireto para a } \\
\text { economia local e/ou regional }\end{array}$ \\
& & $\begin{array}{l}\text { Geração de empregos locais/regionais } \\
\text { Desenvolvimento ou difusão local da } \\
\text { tecnologia importada }\end{array}$ \\
& & $\begin{array}{l}\text { Investimento em infraestrutura } \\
\text { local/regional }\end{array}$ \\
\hline
\end{tabular}


Tabela 1 (continuação)

\begin{tabular}{|c|c|c|c|}
\hline Conceito & Dimensão & Componente & Indicadores \\
\hline & & Proteção Ambiental & Utilização eficiente dos recursos naturais \\
\hline & & & $\begin{array}{l}\text { Redução de ruído, odores, poeira ou } \\
\text { poluentes }\end{array}$ \\
\hline & & & $\begin{array}{l}\text { Melhoria e/ou proteção dos recursos } \\
\text { naturais }\end{array}$ \\
\hline & & & Melhoria na utilização de energia \\
\hline & & & Promoção de energias renováveis \\
\hline & & $\begin{array}{l}\text { Desenvolvimento } \\
\text { Social }\end{array}$ & $\begin{array}{l}\text { Melhoria das condições de trabalho e/ou } \\
\text { direitos humanos }\end{array}$ \\
\hline & & & Promoção da educação \\
\hline & & & $\begin{array}{l}\text { Melhoria das condições de saúde e } \\
\text { segurança }\end{array}$ \\
\hline & & & Redução da pobreza \\
\hline & & & Envolvimento da Comunidade Local \\
\hline & & & $\begin{array}{l}\text { Empoderamento das mulheres, o cuidado } \\
\text { das crianças e dos vulneráveis }\end{array}$ \\
\hline
\end{tabular}

Nota. Fonte: United Nations Framework Conventions on Climate Change. (2011). Benefits of the clean development mechanism 2011. Retrieved from https://cdm.unfccc.int/about/dev_ben/ABC_2011.pdf

Uma segunda fase envolveu a construção de um banco de dados com as informações dos 170 projetos brasileiros registrados no MV, até dezembro de 2013, que foram mapeados na primeira fase do trabalho e também a seleção de alguns para a realização de estudo de casos. Nessa segunda fase da pesquisa foram escolhidos quatro deles que representassem a diversidade dos projetos estudados e, para tanto, os critérios se basearam em: (a) diferentes Padrões de Certificação; (b) diferentes escopos setoriais; (c) viabilidade de visitas de campo (limitações foram impostas pela localização geográfica e dificuldade de acesso, bem como aceitação e interesse dos gestores); (d) número de projetos por escopo (foram priorizados os escopos com maior número, não sendo escolhidos projetos dos padrões de certificação CCX e ACR, uma vez que contêm nove e duas iniciativas, respectivamente). Desta forma, os casos ficaram assim distribuídos (Tabela 2):

Tabela 2

Projetos Selecionados para Realização de Estudos de Casos Ilustrativos

\begin{tabular}{ccc}
\hline Padrão de Certificação & Total de Projetos & Projeto \\
\hline VCS & 108 & Nobrecel \\
Social Carbon & 44 & Cerâmica Santa Izabel \\
CCB & 05 & Corredor Monte Pascoal-Pau Brasil \\
CCX & 09 & Fogões Eficientes \\
Gold Standard & 01 & - \\
Swiss Standard & 01 & - \\
ACR & 02 & - \\
\hline
\end{tabular}

Nota. Fonte: Elaborado a partir de dados extraídos dos DCP constantes no Markit Environmental Registry (n.d.). Registry Public view. Recuperado de https://mer.markit.com/br-reg/public/index.jsp?s=cp 
A terceira fase abrangeu a realização dos quatro estudos de caso ilustrativos envolvendo a triangulação/confrontação entre os dados secundários coletados na primeira fase e os dados primários coletados através de visitas de campo aos projetos selecionados (realizadas no período março de 2012 a dezembro de 2013); observação direta dos pesquisadores (com anotações em diário de campo) e entrevistas semiestruturadas (com gravação de áudio) com gestores, funcionários, técnicos, colaboradores e membros da comunidade local dos projetos visitados. A escolha dos entrevistados foi determinada pela relevância dos mesmos no processo de implantação/operação dos projetos, proximidade com as atividades desenvolvidas, conhecimento da realidade estudada, assim como a disponibilidade dos mesmos para a realização das entrevistas. Desta forma, toda a terceira fase do procedimento metodológico foi considerada como análise ex post.

Assim, a interpretação dos dados/resultados de cada estudo de caso se deu a partir da triangulação/confrontação dos dados secundários recolhidos na primeira fase da pesquisa com os dados primários recolhidos nas visitas de campo, observação direta e entrevistas, tendo como base os indicadores do framework da pesquisa baseados no UNFCCC (2011).

A cada indicador foi atribuído um parâmetro a ser associado segundo sua ocorrência $(+1)$, ausência (0) ou manifestação negativa (-1) na análise documental (análise ex ante) e na feita durante a realização dos estudos de caso (análise ex post).

Dessa forma, cinco pontuações distintas sobre a ocorrência de cobenefícios estão apresentadas na Tabela 3:

Tabela 3

\section{Pontuação dos Cobenefícios}

\begin{tabular}{lc}
\hline Situação & Pontuação \\
\hline Não declarado na análise ex ante, nem visualizado na análise ex post & 0 \\
Visualizado positivamente na análise ex post, mas não declarado na análise ex ante & +1 \\
Declarado positivamente na análise ex ante, mas não visualizado análise ex post & +1 \\
Visualizado negativamente na análise ex post, mas não declarado na análise ex ante & -1 \\
Declarado negativamente na análise ex ante, mas não visualizado na análise ex post & -1 \\
\hline
\end{tabular}

Nota. Fonte: Elaborado pelos autores.

\section{Resultados e Discussões}

Para melhor compreensão do estudo, inicialmente, apresenta-se breve descrição dos quatro casos (Tabela 4): 
Tabela 4

Breve Caracterização dos Casos Analisados

\begin{tabular}{llllll}
\hline Projeto & Localização & $\begin{array}{l}\text { Ano } \\
\text { Início }\end{array}$ & de Caracterização & CertificadoresPC & Breve Descritivo \\
\hline $\begin{array}{l}\text { Corredor } \\
\text { Ecológico }\end{array}$ & Itabela (BA) & 2008 & $\begin{array}{l}\text { Conservação e } \\
\text { Reflorestamento }\end{array}$ & $\begin{array}{l}\text { Climate, Community } \\
\text { and Biodiversity } \\
\text { (CCB) }\end{array}$ & $\begin{array}{l}\text { Desenvolvedores: representantes e } \\
\text { membros da comunidade local e } \\
\text { Pascoal Pau }\end{array}$ \\
& & & & $\begin{array}{l}\text { ONGs socioambientais. } \\
\text { Restauração de mil hectares de }\end{array}$
\end{tabular}

Brasil

(CEMPPB)

Fogões

Eficientes
Maragogipe (BA) 2011

(2011

Cerâmica Itaboraí (RJ)

Santa Izabel

\author{
Substituição de Gold Standard \\ Fogões \\ Rudimentares por \\ Mais Eficientes
}

Troca de

Combustível

Fóssil em

Indústria de

Cerâmica

\section{Verified Carbon Standard (VCS) + Social Carbon}

Verified Carbon Standard (VCS)

Combustível

Fóssil em

Indústria de

Celulose com

cogeração de energia mata nativa para formação de corredores ecológicos ligando os parques nacionais Pau Brasil e Monte Pascoal. As sementes são coletadas e as mudas cultivadas por membros da comunidade local. Investimento em capacitação técnica para manejo sustentável dos recursos.

Desenvolvedores: representantes e membros da comunidade local e ONGs socioambientais.

Substituição de mil fogões rudimentares movidos à lenha por outros mais eficientes. Prevê redução do consumo de madeira (cerca de 50\%) e de emissão de GEE, com melhorias significativas à saúde. Envolvimento de agentes locais para a construção e manutenção dos fogões, bem como para o estabelecimento de relações com os interessados.

Desenvolvedores: Indústria de cerâmica e Empresa de consultoria.

Integra um Programa de Atividades (PoA) com outros dois projetos nas circunvizinhas Guaraí e Itabira, dada a inviabilidade econômica, ambiental e social de um projeto isolado na região. A atividade do projeto consiste na substituição de combustível fóssil (lenha) por biomassa. Envolvimento dos trabalhadores com melhoria de suas condições de trabalho e vida.

Desenvolvedores: Indústria de celulose e papel e empresa de consultoria.

Geração de energia renovável através da instalação de uma nova caldeira de biomassa e uma nova turbina, gerando eletricidade a partir do vapor em alta pressão. A energia é aproveitada no processo de produção.

Nota. Fonte: Elaborado pelos autores com base em seus DCP constantes no Markit Environmental Registry (n.d.). Registry Public view. Recuperado de https://mer.markit.com/br-reg/public/index.jsp?s=cp 
Nessas iniciativas, diferentes contornos dos cobenefícios ambientais, sociais e econômicos foram encontrados, o que pode ser inicialmente explicado por suas diferentes características e escopos de atividade (Tabela 5), entre outros pontos explorados adiante.

Tabela 5

Cobenefícios dos Projetos Analisados

\begin{tabular}{|c|c|c|c|c|c|}
\hline Dimensão & Cobenefício & СЕМРPB & $\begin{array}{c}\text { Fogões } \\
\text { Eficientes }\end{array}$ & $\begin{array}{c}\text { Cerâmica } \\
\text { Santa Izabel }\end{array}$ & Nobrecel \\
\hline \multirow[t]{4}{*}{ Econômica } & $\begin{array}{l}\text { Benefício financeiro direto/indireto } \\
\text { para a economia local e/ou regional }\end{array}$ & +1 & +1 & 0 & +1 \\
\hline & $\begin{array}{l}\text { Geração de empregos } \\
\text { locais/regionais }\end{array}$ & +1 & +1 & +1 & +1 \\
\hline & $\begin{array}{l}\text { Desenvolvimento ou difusão local da } \\
\text { tecnologia importada }\end{array}$ & +1 & +1 & 0 & 0 \\
\hline & $\begin{array}{l}\text { Investimento em infraestrutura } \\
\text { local/regional }\end{array}$ & 0 & +1 & 0 & 0 \\
\hline \multirow[t]{5}{*}{ Ambiental } & $\begin{array}{l}\text { Utilização eficiente dos recursos } \\
\text { naturais }\end{array}$ & +1 & +1 & +1 & +1 \\
\hline & $\begin{array}{l}\text { Redução de ruído, odores, poeira ou } \\
\text { poluentes }\end{array}$ & 0 & +1 & 0 & 0 \\
\hline & $\begin{array}{l}\text { Melhoria e/ou proteção dos recursos } \\
\text { naturais }\end{array}$ & +1 & +1 & 0 & 0 \\
\hline & Melhoria na utilização de energia & 0 & 0 & +1 & +1 \\
\hline & Promoção de energias renováveis & 0 & 0 & +1 & 0 \\
\hline \multirow[t]{6}{*}{ Social } & $\begin{array}{l}\text { Melhoria das condições de trabalho } \\
\text { e/ou direitos humanos }\end{array}$ & 0 & +1 & +1 & +1 \\
\hline & Promoção da educação & +1 & 0 & +1 & 0 \\
\hline & $\begin{array}{l}\text { Melhoria das condições de saúde e } \\
\text { segurança }\end{array}$ & 0 & +1 & +1 & +1 \\
\hline & Redução da pobreza & +1 & +1 & 0 & 0 \\
\hline & Envolvimento da comunidade local & +1 & +1 & +1 & 0 \\
\hline & $\begin{array}{l}\text { Empoderamento das mulheres, o } \\
\text { cuidado das crianças e dos } \\
\text { vulneráveis }\end{array}$ & 0 & +1 & 0 & 0 \\
\hline
\end{tabular}

Nota. Fonte: Elaborado pelos autores a partir das análises ex ante e ex post dos relatórios de auditoria, corporativos e técnicos relacionados aos Documentos de Concepção dos Projetos (DCP) disponíveis no Markit Environmental Registry (n.d.). Registry - Public view. Recuperado de https://mer.markit.com/br-reg/public/index.jsp?s=cp

Na dimensão econômica, considerou-se como importada aquela tecnologia não presente na comunidade ou de seu domínio e conhecimento, o que não significa necessariamente que seja proveniente de outro país.

O Corredor Ecológico Monte Pascoal - Pau Brasil (CEMPPB) foi o primeiro projeto da América Latina de restauração florestal registrado no CCB. Localizado em uma região de mata atlântica sob forte pressão antrópica, o CEMPPB objetiva formar ou reconstituir corredores ecológicos ligando dois Parques Nacionais (Pau Brasil e Monte Pascoal). Com duração prevista de 30 anos, articula membros da comunidade local e ONGs socioamentais no intuito de reduzir 316 mil $\mathrm{tCO}_{2 \mathrm{eq}}$ (pequena 
escala) a partir do plantio de espécies nativas originadas da coleta de sementes e de mudas cultivadas por atores locais, previamente capacitados em técnicas de reflorestamento.

O projeto declara em seus documentos, sendo confirmados com a visita, atingir oito cobenefícios locais em todas as três dimensões investigadas (com maior destaque para o econômico e social), conforme consta na Tabela 5.

O projeto Fogões Eficientes consiste na substituição de mil dos tradicionais fogões à lenha utilizados na região por fogões melhorados que permitem o uso mais eficiente da madeira e, portanto, reduzem o seu consumo em escala local e as emissões de $\mathrm{CO}_{2}$. Ele foi concebido e implementado pela ONG Instituto Perene, com forte apoio da comunidade local. Os créditos de carbono do projeto são todos vendidos antecipadamente para a empresa Natura, que pretende utilizá-los, por meio de um programa interno (carbono neutro), como forma de compensar as suas emissões de GEE. Todo o recurso recebido com a venda dos créditos está sendo reinvestido no próprio projeto, desse modo, beneficiando diretamente a comunidade local.

Esta iniciativa foi validada pela metodologia da Gold Standard, a qual requer envolvimento dos agentes locais (comunidades rurais) desde as primeiras fases de concepção do projeto, constituindo-se um fator fundamental para a implantação do mesmo. Assim, foram constatados cobenefícios em escala local que vão além da redução de emissão de GEE. Dos 15 indicadores estabelecidos, o projeto contribui positivamente para 12, conforme pode ser observado na Tabela 5 e descrito na Tabela 6 . Ademais, as dimensões ambiental e social constituíram-se como as de maior impacto positivo.

Diferentemente dos cenários positivos anteriormente analisados, o projeto da Cerâmica Santa Izabel apresentou contribuição apenas mediana para o DS, já que impactou positivamente em oito dos 15 indicadores elencados, discriminados na Tabela 5. O projeto faz parte de um programa de atividades denominado Programa de Atividades (PoA, em sua sigla em inglês Programme of Activities), juntamente com outros dois projetos de cerâmica - Guaraí e Itabira. Esta união de projetos em um PoA fez-se necessária porque os projetos, isoladamente, não são viáveis em termos econômicos, ambientais e sociais. Tal iniciativa consiste em utilizar madeira de florestamento e resíduos lenhosos (como aparas de madeira), que são biomassas renováveis, para alimentar os fornos em vez de usar um combustível não renovável como o petróleo pesado. Ressalta-se que um PoA é definido pela Comissão Interministerial de Mudança Global do Clima como:

uma ação coordenada voluntária adotada por uma entidade privada ou pública que coordena e executa qualquer política/medida ou meta definida (isto é, esquemas de incentivo e programas voluntários) que acarretem reduções das emissões ... que sejam adicionais as que ocorreriam na ausência do programa de atividades, por meio de um número ilimitado de atividades programáticas (Resolução n. 9, 2009, p. 13).

O projeto Nobrecel consiste na geração de energia renovável através da instalação de uma nova caldeira de biomassa e uma nova turbina. Trata-se de um projeto de cogeração desenvolvido por empresa privada que utiliza resíduos de biomassa como combustível. Assim como o projeto de Cerâmica, verificou-se pouca contribuição para o DS nacional e/ou local, registrando apenas impacto em seis dos 15 indicadores (ver Tabela 5).

Os principais resultados encontrados nos quatro estudos de caso estão apresentados na Tabela 6. Vale notar que, para todas as três dimensões, muitos dos impactos identificados são especialmente relevantes em âmbito local, de modo que é possível inferir que projetos brasileiros de mitigação registrados no mercado voluntário possuem um grande potencial para promover o desenvolvimento sustentável local. 
Tabela 6

\section{Descritivo Comparativo das Contribuições dos Projetos Analisados por Dimensão}

\begin{tabular}{|c|c|c|c|}
\hline Projeto & Dimensão Econômica & Dimensão Ambiental & Dimensão Social \\
\hline $\begin{array}{l}\text { Corredor } \\
\text { Ecológico } \\
\text { Monte } \\
\text { Pascoal - Pau } \\
\text { Brasil }\end{array}$ & $\begin{array}{l}\text { Oportunidades de trabalho e } \\
\text { geração de renda locais } \\
\text { associadas à recuperação } \\
\text { ambiental (troca de } \\
\text { atividade predatório como } \\
\text { supressão ilegal de mata } \\
\text { nativa, modalidades de } \\
\text { pesca irregular por ofícios } \\
\text { de restauração florestal, } \\
\text { como coleta e cultivo de } \\
\text { sementes e plantio de } \\
\text { mudas nativas, manutenção } \\
\text { e monitoramento da mata). }\end{array}$ & $\begin{array}{l}\text { Constatados benefícios relacionados à } \\
\text { manutenção e restauração de serviços } \\
\text { ecossistêmicos diversos que } \\
\text { influenciam no desenvolvimento } \\
\text { sustentável nacional e local (como a } \\
\text { comuns a outros projetos de } \\
\text { reflorestamento com espécies nativas, } \\
\text { segundo Stickler et al., 2009). } \\
\text { Verificadas alterações ambientais } \\
\text { positivas locais, como a diminuição da } \\
\text { erosão do solo, melhora na oferta } \\
\text { natural de recursos hídricos e menor } \\
\text { incidência de pragas em lavouras, dado } \\
\text { o maior equilíbrio ecossistêmico. Esse } \\
\text { cenário resultou na redução do uso de } \\
\text { meios artificais para controle de pragas } \\
\text { (pesticidas e outros agrotóxicos) e para } \\
\text { restauração da qualidade do solo nos } \\
\text { cultivos comerciais, diminuindo custos } \\
\text { com manutenção da lavoura. Também }\end{array}$ & $\begin{array}{l}\text { Destaque para o envolvimento } \\
\text { comunitário no desenvolvimento das } \\
\text { atividades, implementado através de } \\
\text { uma rede institucional formada por } \\
\text { ONGs, associações e cooperativas } \\
\text { locais, produtores rurais e moradores. } \\
\text { Este arranjo almeja promover uma } \\
\text { governança includente e participativa, } \\
\text { desenvolvendo ações voltadas ao } \\
\text { empoderamento local e formação de } \\
\text { capital humano (como a capacitação } \\
\text { técnica de moradores para manejo } \\
\text { sustentável dos recursos florestais), o } \\
\text { que é destacado na literatura para o } \\
\text { sucesso e permanência dos projetos de } \\
\text { carbono florestal (Colfer, 2011; Larson } \\
\text { \& Petkova, 2011). Apontado o } \\
\text { fortalecimento e fomento de } \\
\text { associativismo local e geração de } \\
\text { empregos e renda diretos. }\end{array}$ \\
\hline
\end{tabular}
foi relatada a reaparição mais corriqueira de espécies de pequenos pássaros e animais caracterísicos da região no passado.

Fogões

Eficientes
Apontada geração de A utilização eficiente dos recursos Destaque para o envolvimento da emprego local, alimentada naturais decorre do bom uso da comunidade local que apoiou na pela opção de aquisição de madeira para queima no fogão implantação e adequação do projeto a insumos para construção (redução média de 50\%). A tecnologia realidade local, a exemplo da altura da dos fogões por fornecedores empregada nos novos fogões eficientes cozinha, que foi modificada para locais. apoia nesse resultado já que evita a satisfazer os interesses de mulheres fuga do calor resultando numa locais. Além disso, observa-se a diminuição da demanda por lenha. presença de uma agente multiplicadora Ademais, a lenha no projeto é "líder", que repassa os treinamentos de composta por galhos e pequenos implantação e manutenção dos fogões troncos, e essa redução reflete na para os demais agentes e para os mitigação do desmatamento e erosão pedreiros. Também os agentes de do solo. saúde local estão envolvidos no A redução de ruído, odores, poeira ou processo, pois, como usuários dos poluentes tem se mostrado uma das fogões, participam de forma bastante mais significativas, principalmente, no agregadora no processo de que se refere à redução da poluição do disseminação da proposta, utilizandoar. Os fogões acabam por reduzir a se de sua fácil penetração na emissão de fumaça dentro das casas e, comunidade local.

por conseguinte, isso corrobora O processo de construção dos fogões positivamente a melhoria no aspecto eficientes pressupõe a participação social. São constatadas melhorias no ampla da comunidade, apoiando a trato das doenças respiratórias formação dos usuários como parte das inferiores, bem como dor nas costas atividades do projeto. Desta forma, (decorrente da ausência da necessidade beneficia-se o usuário final em termos de não ter que carregar tanta madeira), de formação e treinamento para assim como nos problemas de visão utilização e manutenção das cozinhas. (devido à diminuição/desaparecimento Tudo isso acarreta em efeitos positivos de fumo).

na promoção do desenvolvimento

Foi também apontada redução no risco sustentável local.

de incêndio e explosões, já que com os novos fogões eficientes é reduzida a quantidade de lenha armazenada. 


\section{Tabela 6 (continuação)}

\begin{tabular}{|c|c|c|c|}
\hline Projeto & Dimensão Econômica & Dimensão Ambiental & Dimensão Social \\
\hline $\begin{array}{l}\text { Cerâmica } \\
\text { Santa Izabel }\end{array}$ & $\begin{array}{l}\text { Na medida em que a injeção } \\
\text { da biomassa nos fornos é } \\
\text { realizada manualmente } \\
\text { pelos operadores, o projeto } \\
\text { acaba por requerer mais } \\
\text { trabalhadores, uma vez que } \\
\text { a madeira deve ser cortada } \\
\text { com um machado } \\
\text { hidráulico e movida ao } \\
\text { longo da cerâmica, } \\
\text { corroborando para geração } \\
\text { de empregos locais. }\end{array}$ & $\begin{array}{l}\text { Constatada utilização eficiente dos } \\
\text { recursos naturais e promoção de } \\
\text { energias renováveis, decorrente da } \\
\text { utilização da biomassa renovável para } \\
\text { alimentar os fornos em substituição a } \\
\text { um combustível não renovável, como } \\
\text { óleo. } \\
\text { Aquisição de novos equipamentos, } \\
\text { como queimadores mecânicos e } \\
\text { termopares, que contribuíram para } \\
\text { melhoria na eficiência energética, pois } \\
\text { minimiza a perda de calor na produção } \\
\text { da cerâmica. Foram também instaladas } \\
\text { estruturas metálicas que capturam } \\
\text { calor do sol para a secagem, tornando- } \\
\text { a mais rápida e natural, não sendo } \\
\text { necessária utilização de ventiladores - } \\
\text { o que diminui o gasto de energia. }\end{array}$ & $\begin{array}{l}\text { Da aquisição dos novos equipamentos } \\
\text { decorreu a capacitação dos } \\
\text { funcionários para o seu manuseio, } \\
\text { tendo sido ministradas palestras } \\
\text { relacionadas à melhoria de vida, o que } \\
\text { contribuiu para certa melhora nas } \\
\text { condições de trabalho, como maior uso } \\
\text { de equipamentos de proteção } \\
\text { individual, com resultados na saúde } \\
\text { geral do indivíduo. }\end{array}$ \\
\hline
\end{tabular}

Nobrecel O projeto constitui uma Verificada redução do uso de Observada melhoraria na condição iniciativa inovadora dentro combustíveis fósseis, substituindo-os ambiental local devido à destinação do setor energético por uma fonte alternativa renovável. adequada de resíduos de serragem e brasileiro e pretende, com Visa também à geração de energia, já lascas de madeira.

isso, contribuir para a que uma turbina instalada As melhorias nas condições de integração regional e complementarmente para a caldeira trabalho e de saúde decorrem da conexão com outros setores, gera eletricidade a partir do vapor de promoção de treinamentos aos como engenharia e alta pressão. Esta energia elétrica é funcionários da empresa que passam a construção civil, utilizada no processo de produção, operar com novo combustível que estimulando cobenefícios bem como em outros processos requer maior cuidado no seu manuseio. financeiros diretos e rotineiros. Foram desenvolvidos cursos de indiretos para a região. $\quad$ formação extensiva sobre manipulação Apontada contribuição com da biomassa e condições mais seguras a geração de emprego e de trabalho, a fim de esclarecer para os renda local, uma vez que empregados os novos procedimentos proporciona novas relacionados com a tecnologia adotada. oportunidades de trabalho. Os recursos auferidos com a venda dos créditos têm sido revertidos em melhorias nos processos internos na organização.

Nota. Fonte: Elaborado pelos autores.

\section{Considerações Finais}

A mitigação das mudanças climáticas pode oferecer aos países não industrializados uma oportunidade de revisitar suas estratégias de desenvolvimento sob uma nova perspectiva (Begg et al., 2002).

No presente artigo, buscou-se identificar e analisar os cobenefícios existentes em projetos de redução de emissão de GEE do MV brasileiro. Assim como o MR, o MV é um mecanismo importante que apoia as políticas de mudanças climáticas. Espera-se, em ambos os mercados, que a implantação dos projetos de mitigação possa contribuir para o DS dos países hospedeiros (UNFCCC, 2011). Este estudo ressalta a importância que o MV tem em auxiliar as comunidades locais a implementarem ações em prol do DS local e verifica a conexão entre esse resultado e as exigências de caráter social de alguns Padrões de Certificação que suplantam as meras exigências de mitigação de GEE. 
Conforme dados apresentados, comparando-se os quatro projetos eleitos como estudo de caso, é possível verificar-se que dois deles apresentam avanços importantes na promoção do DS. Estes são os projetos em que se verificou uma maior participação de atores sociais diversos, além de estarem atrelados a Padrões de Certificação que estipulam regras claras para cobenefícios a serem trazidos pelos projetos. A constatação de efetiva contribuição de projetos de MV ao DS vem ao encontro de resultados anteriores de pesquisas em estudos de caso único (a exemplo de Paiva, Gomes, Fernández, \& Andrade, 2014; Ventura, Fernández, Andrade, \& Lumbreras, 2012) e contradiz os resultados da UNFCCC (2011) e Boyd et al. (2009) nos seus estudos sobre o MR. O resultado alcançado confirma o ponto de partida deste estudo sobre o maior potencial do MV para contribuição ao DS, dada sua maior flexibilidade, diversidade de atores e exigência por parte de alguns Padrões de Certificação no atendimento à cobenefícios específicos, corroborando os estudos de Crowe (2013), em que ressalta o papel dos diversos atores e padrões no alcance dos cobenefícios desejados.

De certa forma, foram apontadas contribuições em todos cobenefícios elencados na pesquisa, sendo os cobenefícios utilização eficiente dos recursos naturais e geração de empregos locais/regionais verificados em todos os projetos. As perspectivas social e econômica apresentaram maior contribuição para o DS para os estudos realizados. Tais resultados diferem dos resultados verificados na análise do UNFCCC (2011), em que a perspectiva social não apresenta resultado significativo.

Alguns cobenefícios estimulam outros, como é o caso da melhoria das condições de trabalho com melhoria nas condições de saúde e segurança. Ademais, os projetos que apresentaram maiores cobenefícios foram registrados nos Padrões de Certificação CCB e Gold Standard, que exigem o atendimento a indicadores ambientais e sociais que vão além da redução de emissão de GEE.

O resultado alcançado neste estudo reforça as prioridades expressas pelo Conselho Executivo do MDL, que permitiu, em 2011, contribuições públicas sobre formas de inclusão dos cobenefícios e diminuição do ciclo de vida e dos custos de transação dos projetos de MDL e o papel dos diferentes intervenientes e interessados neste processo. Este artigo se propôs a realizar uma contribuição diferente da maioria dos artigos científicos da área ao contemplar, além da análise documental, a realização de quatro estudos de caso no Brasil, realizando triangulação dos dados para tanto. Ainda que não se possa extrapolar a análise para todo o mercado de carbono voluntário brasileiro, a pesquisa revela avanços no conhecimento sobre a contribuição dos projetos estudados na geração de cobenefícios ao desenvolvimento sustentável para além da redução de GEE.

\section{Referências}

Alexeew, J., Bergset, L., Meyer, K., Peterson, J., Schneider, L., \& Unger, C. (2010). An analysis of the relationship between the additionality of CDM projects and their contribution to sustainable development. International Environmental Agreements: Politics, Law and Economics, 10(3), 233-248. Retrieved

from http://www.kfe.uzh.ch/teaching/KfEKonferenz/Text\%20Workshop\%20Stadelmann.pdf. doi: 10.1007/s10784-010-9121-y

Anderson, S. (2011, agosto). Cambio climático y reducción de la pobreza (Informe de Política). Alianza Clima y Desarrollo. Recuperado de http://cdkn.org/wp-content/uploads/2012/03/Policybrief_CC-Reduccion-de-la-Pobreza.pdf

Andrew, A. M. (2011). Second thoughts on global warming. Kybernetes, 40(1/2), 327-329. Retrieved from http://www.emeraldinsight.com/journals.htm?articleid=1921821

Bayon, R., Hawn, A., \& Hamilton, K. (2009). Voluntary carbon markets: an international business guide to what they are and how they work (2nd ed.). London: Earthscan. 
Begg, K., Parkinson, S., vd Horst, D., Wilkinson, R., Theuri, D., Gitonga, S., Mathenga, M., AmissahArthur, H., Atugba, S., \& Ackon, S. (2003). Encouraging CDM energy projects to aid poverty alleviation (Final report of project R8037 under the DFID KAR programme). Retrieved from http://www.iesd.dmu.ac.uk/contract_research/publications/kb1.pdf

Benessaiah, K. (2012). Carbon and livelihoods in Post-Kyoto: assessing voluntary carbon markets. Ecological Economics, $77 . \quad 1-6 . \quad$ Retrieved from http://www.sciencedirect.com/science/article/pii/S0921800912000869\#. doi: 10.1016/j.ecolecon.2012.02.022

Blois, J., Zarnetske, P. L., Fitzpatrick, M. C., \& Finnegan, S. (2013). Climate change and the past, present, and future of biotic interactions. Science, 341(6145), 499-504. Retrieved from http://www.sciencemag.org/content/341/6145/499.full.html. doi: 10.1126/science.1237184

Boyd, E., Hultman, N., Roberts, J., Corbera, E., Cole, J., Bozmoski, A., Ebeling, J., Tippman, R., Mann, P., Brown, K., \& Liverman, D. M. (2009). Reforming the CDM for sustainable development: lessons learned and policy futures. Enviromental Science \& Policy, 12(7), 820-831. doi: 10.1016/j.envsci.2009.06.007

Bozmoski, A., Lemos, M. C., \& Boyd, E. (2008). Prosperous negligence: governing the clean development mechanism for markets and development. Environment, 50(3), 18-30. doi: 10.3200/ENVT.50.3.18-30

Bumpus, A. G., \& Cole, J. C. (2010). How can the current CDM deliver sustainable development? Wiley Interdisciplinary Reviews: Climate Change, 1(4), 541-547. doi: 10.1002/wcc.57

Capoor, K., \& Ambrosi, P. (2006). State and trends of the carbon market. Retrieved from https://wbcarbonfinance.org/docs/StateoftheCarbonMarket2006.pdf

Castro, P., \& Michaelowa, A. (2010). The impact of discounting emission credits on the competitiveness of different CDM host countries. Ecological Economics, 70(1), 34-42. doi: 10.1016/j.ecolecon.2010.03.022

Colfer, C. J. P. (2011). Marginalized forest peoples' perceptions of the legitimacy of governance: an exploration. World Development, 39(12), 2147-2164. doi: 10.1016/j.worlddev.2011.04.012

Corbera, E., Estrada, M., \& Brown, K. (2009). How do regulated and voluntary carbon-offset schemes compare?. Journal of Integrative Environmental Sciences, 6(1), 25-50. doi: $10.1080 / 15693430802703958$

Cosbey, A., Parry, J.-E., Browne, J., Babu, Y. D., Bhandari, P., Drexhage, J., \& Murphy, D. (2005). Realizing the development dividend: making the CDM work for developing countries (Phase 1 Report-Prepublication Version). International Institute for Sustainable Development (IISD). Retrieved from http://www.iisd.org/sites/default/files/pdf/2005/climate_realizing_dividend.pdf

Crowe, T. L. (2013). The potential of the CDM to deliver pro-poor benefits. Climate Policy, 13(1), 5879. doi: $10.1080 / 14693062.2012 .709080$

Drupp, M. A. (2011). Does the gold standard label hold its promise in delivering higher sustainable development benefits? A multi-criteria comparison of CDM projects. Energy Policy, 39(3), 12131227. doi: 10.1016/j.enpol.2010.11.049

Fernández, L. (2014). Evaluación de los co-beneficios sobre el desarrollo sostenible y la reducción de la pobreza de proyectos de mitigación del cambio climático en Brasil (Tesis doctoral). Madrid: Escuela Técnica Superior de Ingenieros Industriales, Universidad Politécnica de Madrid (UPM), Madrid, Espanha. 
Fernández, L., Lumbreras, J., Borge, R., \& Cobo-Benita, J. R. (2011, October). Exploring co-benefits of clean development mechanism (CDM) projects. Proceedings of Conference on Sustainable Development, Shangai, China, 2.

Fernández, L., Sota, C. de la, Andrade, J. C. S., Lumbreras, J., \& Mazorra, J. (2014). Social development benefits of hydroelectricity CDM projects in Brazil. International Journal of Sustainable Development \& World Ecology, 21(3), 246-258. doi: 10.1080/13504509.2014.909901

Guigon, P., Bellassen, V., \& Ambrosi, P. (2009). Voluntary carbon markets: what the standard say [Working Paper $\mathrm{n}^{\circ}$ 2009-4]. Mission Climat. Retrieved from http://www.indiaenvironmentportal.org.in/files/voluntary_carbon_marketswhat_the_standards_say.pdf

Harris, E. (2007). The voluntary carbon offsets market: an analysis of market characteristics and opportunities for sustainable development. International Institute for Environment and Development, London. Retrieved from http://pubs.iied.org/pdfs/15507IIED.pdf

Hoffman, A. J. (2004). Climate change strategy: the business logic behind voluntary greenhouse gas reductions [Working Paper $\mathrm{N}^{\mathrm{a}}$ 905]. University of Michigan, Ann Arbor, MI, USA. Retrieved from http://deepblue.lib.umich.edu/bitstream/handle/2027.42/39160/905.pdf?sequence=1

Intergovernmental Panel on Climate Change. (2013). Summary for policymakers. Retrieved from http://www.climatechange2013.org/images/report/WG1AR5_SPM_FINAL.pdf

Jung, M. (2006). Host country attractiveness for CDM non-sink projects. Energy Policy, 34(15), $2173-$ 2184. doi: 10.1016/j.enpol.2005.03.014

Kollmuss, A., Zink, H., \& Polycarp, C. (2008). Making sense of the voluntary carbon market: a comparison of carbon offset standards. Retrieved from assets.panda.org/downloads/vcm_report_final.pdf

Larson, A. M., \& Petkova, E. (2011). An introduction to forest governance, people and REDD+ in Latin America: obstacles and opportunities. Forests, 2(1), 86-111. doi: 10.3390/f2010086

Mackerron, G. J., Egerton, C., Gaskell, C., Parpia, A., \& Mourato, S. (2009). Willingness to pay for carbon offset certification and co-benefits among (high-) flying young adults in the UK. Energy Policy, 37(4), 1372-1381. Retrieved from http://www.sciencedirect.com/science/article/pii/S0301421508007179\#. doi: 10.1016/j.enpol.2008.11.023

Markit Environmental Registry (n.d.). Registry - Public view. Retrieved from https://mer.markit.com/brreg/public/index.jsp?s=cp.

Merger, E., Dutschke, M., \& Verchot, L. (2011). Options for REDD+ voluntary certification to ensure net GHG benefits, poverty alleviation, sustainable management of forests and biodiversity conservation. Forests, 2(2), 550-577. Retrieved from http://www.mdpi.com/1999-4907/2/2/550. doi: $10.3390 / f 2020550$

Ministério da Ciência e Tecnologia. (2011). Status atual das atividades de projeto no âmbito do mecanismo de desenvolvimento limpo (MDL) no Brasil e no mundo. Recuperado de http://www.mct.gov.br/upd_blob/0215/215908.pdf

Miyatsuka, A., \& Zusman, E. (2012). Fact sheet $n^{o}$ 1: what are co-benefits? Asian Co-benefits Partnership. 
Newell, P., Jenner, N., \& Baker, L. (2009). Governing clean development: a framework for analysis [Working Paper $\mathrm{n}^{\mathrm{o}}$ 001]. Governance of Clean Development. Retrieved from http://www.tyndall.ac.uk/sites/default/files/GCD_WorkingPaper001.pdf

Nussbaumer, P. (2009). On the contribution of labelled certified emission reductions to sustainable development: a multi-criteria evaluation of CDM projects. Energy Policy, 37(1), 91-101. doi: 10.1016/j.enpol.2008.07.033

Olhoff, A., Markandya, A., Halsnaes, K., \& Taylor, T. (2004). CDM sustainable development impacts. Roskilde, Denmark: UNEP. Retrieved from http://cd4cdm.org/publications/cdm\%20sustainable\%20development\%20impacts.pdf

Olsen, K. H. (2007). The clean development mechanism's contribution to sustainable development: a review of the literature. Climatic Change, 84(1), 59-73. Retrieved from http://www.springerlink.com/content/60g30h3367115396/. doi: 10.1007/s10584-007-9267-y

Olsen, K. H., \& Fenhann, J. (2008). Sustainable development benefits of clean development mechanism projects: a new methodology for sustainability assessment based on text analysis of the project design documents submitted for validation. Energy Policy, 36(8), 2819-2830. doi: 10.1016/j.enpol.2008.02.039

Orford, M., Raubenheimer, S., \& Kantor, B. (2004). Climate change and the Kyoto protocol's clean development mechanism. London: ITDG Publishing.

Organização das Nações Unidas. (2012). Convenção sobre mudança do clima: o Brasil e a convençãoquadro das nações unidas. Brasília: MCT. Recuperado de http://www.onu.org.br/rio20/img/2012/01/convencao_clima.pdf

Overseas Environmental Cooperation Center. (2009). The co-benefits approach for GHG emission reduction projects. Tokyo, Japan: Ministry of the Environment. Retrieved from http://www.env.go.jp/en/earth/ets/icbaghserp091125.pdf

Paiva, D. S., Gomes, G. A. M. D. M., Fernández, L., \& Andrade, J. C. S. (2014). Voluntary carbon market and its contributions to sustainable development: analysis of the Monte Pascoal-Pau Brazil ecological corridor. International Journal of Innovation and Sustainable Development, 8(1), 116. doi: 10.1504/IJISD.2014.059219

Peters-Stanley, M., \& Yin, D. (2013). Maneuvering the mosaic: state of the voluntary carbon markets 2013. Retrieved from http://www.forest-trends.org/documents/files/doc_3898.pdf

Priem, H. N. A. (2013). Climate change and carbon dioxide: geological perspective. Energy \& Environment, 24(3/4), 361-380. Retrieved from http://multiscience.metapress.com/content/2k8t55012gg587v8/. doi: 10.1260/0958-305X.24.3-4.361

Resolução n. 9, de 20 de março de 2009. (2009). Dispõe sobre o programa de atividades no âmbito do mecanismo de desenvolvimento limpo. Recuperado de: http://www.ambiente.sp.gov.br/biogas/files/2013/12/resolucao_n9_20_marco_2009.pdf

Scafetta, N. (2010). Empirical evidence for a celestial origin of the climate oscillations and its implications. Journal of Atmospheric and Solar-Terrestrial Physics, 72(13), 951-970. Retrieved from http://www.sciencedirect.com/science/article/pii/S1364682610001495. doi: 10.1016/j.jastp.2010.04.015

Seroa da Motta, R., Hargrave, J., Luedemann, G., \& Gutierrez, M. B. S. (Eds.). (2011). Mudança do clima no Brasil: aspectos econômicos, sociais e regulatórios. Brasília: Ipea.

Simoni, W. (2009). Mercado de carbono. In M. A. Fujihara \& F. G. Lopes, Sustentabilidade e mudanças climáticas: guia para o amanhã (pp. 25-86). São Paulo: Editora Senac. 
Soon, W. H. (2007). Implications of the secondary role of carbon dioxide and methane forcing in climate change: past, present, and future. Physical Geography, 28(2), 97-125. Retrieved from http://www.tandfonline.com/doi/abs/10.2747/.U42sBHKSz_E\#.U42u2HKSz_E. doi: 10.2747/0272-3646.28.2.97

Souza, A., Paiva, D., \& Andrade, J. (2011, dezembro). Perfil do mercado voluntário. Anais do Encontro Nacional de Gestão Empresarial e Meio Ambiente, São Paulo, SP, Brasil, 13.

Stickler, C. M., Nepstad, D. C., Coe, M. T., McGrath, D. G., Rodrigues, H. O., Walker, W. S., SoaresFilho, B. S., \& Davidson, E. A. (2009). The potential ecological costs and cobenefits of REDD: a critical review and case study from the Amazon region. Global Change Biology, 15(12), 28032824. doi: 10.1111/j.1365-2486.2009.02109.x

Subbarao, S., \& Lloyd, B. (2011). Can the clean development mechanism (CDM) deliver? Energy Policy, 39(3), 1600-1611. doi: 10.1016/j.enpol.2010.12.036

Sutter, C., \& Parreño, J. C. (2007). Does the current clean development mechanism (CDM) deliver its sustainable development claim? An analysis of officially registered CDM projects. Climatic Change, 84(1), 75-90. doi: 10.1007/s10584-007-9269-9

Taiyab, N. (2005). The market for voluntary carbon offsets: a new tool for sustainable development? [Getpeeker Series n. 121]. International Institute for Environment and Development, London, UK. Retrieved from http://pubs.iied.org/pdfs/14513IIED.pdf

Thomson Reuters. (2013, March 25). Carbon 2013: at a tipping point. 2013. Retrieved from http://www.pointcarbon.com/research/promo/research/1.2236309?\&ref=searchlist

United Nations Framework Conventions on Climate Change. (2011). Benefits of the clean development mechanism 2011. Retrieved from https://cdm.unfccc.int/about/dev_ben/ABC_2011.pdf

Ventura, A. C., Fernández, L., Andrade, J. C., \& Lumbreras, J. (2012). The human side of social technology for climate change mitigation and human development: the case of "fogões ecológicos" in Brazil. The International Journal of Environment and Sustainable Development, 11(4), 375-393. doi: 10.1504

Wood, R. G. (2011). Carbon finance and pro-poor co-benefits: the gold standard and climate, community and biodiversity standards [Discussion paper, n. 4]. London: International Institute for Environment and Development. Retrieved from http://pubs.iied.org/pdfs/15521IIED.pdf

\title{
Dados dos Autores
}

\author{
Danielle Soares Paiva \\ Avenida Reitor Miguel Calmon, s/n., Vale do Canela, 40110-100, Salvador, BA, Brasil. E-mail: paivadani@hotmail.com \\ Luz Garcia Fernandez \\ Calle José Gutiérrez Abascal, 2, 28006, Madrid, Espanha. E-mail: 1fernandezg@etsii.upm.es \\ Andréa Cardoso Ventura \\ Avenida Reitor Miguel Calmon, s/n., Vale do Canela, 40110-100, Salvador, BA, Brasil. E-mail: andreaventurassa@ gmail.com \\ Guineverre Alvarez \\ Avenida Reitor Miguel Calmon, s/n., Vale do Canela, 40110-100, Salvador, BA, Brasil. E-mail: guineverre2@hotmail.com \\ José Célio Silveira Andrade \\ Avenida Reitor Miguel Calmon, s/n., Vale do Canela, 40110-100, Salvador, BA, Brasil. E-mail: jcelio.andrade@gmail.com
}

